\title{
Large-diameter compression arteries as a possible facilitating factor for trigeminal neuralgia: analysis of axial and radial diffusivity
}

\author{
Wei Lin ${ }^{1}$ • Wan-ping Zhu ${ }^{2}$ • Yi-li Chen ${ }^{3}$ • Guo-can Han ${ }^{1} \cdot$ Yue Rong ${ }^{1}$ - Yu-rong Zhou ${ }^{1}$. \\ Qiao-wei Zhang ${ }^{1}$
}

Received: 11 September 2015 / Accepted: 14 December 2015 / Published online: 6 January 2016

(C) The Author(s) 2016. This article is published with open access at Springerlink.com

\begin{abstract}
Background Neurovascular compression (NVC) of the trigeminal nerve is associated with trigeminal neuralgia (TN). Some arteries that compress the trigeminal nerve are large, while others are small. This study evaluated the influence of diameter of compression arteries (DCA) on NVC with and without TN using axial diffusivity (AD) and radial diffusivity (RD) of magnetic resonance (MR) imaging.

Methods Fifty TN patients with unilateral NVC, 50 asymptomatic patients with unilateral $\mathrm{NVC}$, and 50 healthy controls (HC) were divided into three groups (NVC with TN, NVC without TN, and HC). The three groups were imaged with a 3.0-T MR system using three-dimensional fast imaging employing steady-state acquisition (3D FIESTA) and diffusion tensor imaging (DTI). We compared the mean size of DCA between NVC with and without TN. The mean values of $A D$ and RD at the site of NVC were compared between the three groups. Correlation analyses were performed between the DCA and the diffusion metrics (AD and $\mathrm{RD}$ ) in NVC patients with and without TN.
\end{abstract}

Wei Lin

linwei7012@aliyun.com

1 Department of Radiology, Sir Run Run Shaw Hospital, School of Medicine, Zhejiang University, 3 Qingchun East Road, Hangzhou 310016, China

2 Operating Room, Sir Run Run Shaw Hospital, School of Medicine, Zhejiang University, Hangzhou, China

3 Department of Neurosurgery, Sir Run Run Shaw Hospital, School of Medicine, Zhejiang University, Hangzhou, China
Results The mean DCA in NVC patients with TN (1.58 $\pm 0.34 \mathrm{~mm})$ was larger than that without TN $(0.89$ $\pm 0.29 \mathrm{~mm}$ ). Compared with NVC without TN and HC, the mean values of RD at the site of NVC with TN were significantly increased; however, no significant changes of $\mathrm{AD}$ were found between the groups. Correlation analysis showed that DCA positively correlated with RD in NVC patients with and without TN $(r=0.830, p=0.000)$. No significant correlation was found between DCA and AD $(r=0.178, p=0.077)$.

Conclusions Larger-diameter compression arteries may increase the chances of $\mathrm{TN}$, and may be a possible facilitating factor for TN.

Keywords Axial diffusivity · Demyelination - Neurovascular compression $\cdot$ Radial diffusivity $\cdot$ Trigeminal neuralgia

\section{Introduction}

Neurovascular compression (NVC) as a cause of trigeminal neuralgia (TN) is a widely accepted mechanism $[3,6]$; however, amongst all the offending arteries, some are big, while others are small. Is the size of the arteries different between NVC with TN and NVC without TN? To our knowledge, there is no study that answers this particular question. In this study, we aimed to evaluate diameter of compression arteries (DCA) between NVC with TN and without TN, and to investigate whether the size of the DCA has any influence on TN, we compared diffusion metrics (axial diffusivity $[\mathrm{AD}]$ and radial diffusivity $[\mathrm{RD}]$ ) between three groups (NVC with $\mathrm{TN}, \mathrm{NVC}$ without TN, and healthy control [HC] subjects). Further, correlation analyses were performed between the DCA and diffusion metrics. 


\section{Materials and methods}

\section{Participants}

The study was conducted between February 2012 and June 2015. We recruited $50 \mathrm{TN}$ patients with unilateral NVC (19 men, 31 women; age range, 52-82 years, mean age, 57.98 \pm 5.46 years). The diagnosis of all primary $\mathrm{TN}$ patients was confirmed in accordance with the diagnostic criteria of typical TN [15] and treated with microvascular decompression (MVD) at the Department of Neurosurgery, Sir Run Run Shaw Hospital. To validate the study, 50 patients with unilateral NVC without TN (20 men, 30 women; age range, 5080 years, mean age, $57.08 \pm 4.76$ years) and $50 \mathrm{HC}$ subjects (21 men, 29 women; age range, 50-66 years, mean age, 56.88 \pm 3.99 years) were also included in the study. Both of these groups came from the health care center of our hospital and underwent magnetic resonance imaging (MRI) for a routine health check-up. Patients with different illnesses, such as arteriovenous malformations, bony anomalies, hemorrhage, ischemia, multiple sclerosis lesions, cysts, any type of intracranial mass, head trauma, head operation, other disorders that might affect central nervous system function, presence of metal implants, and patient motion during examinations, were excluded. Only healthy controls showing no signs of neurovascular conflict were included. Low-quality images or images containing artifacts were also excluded. Written informed consent was obtained from each participant before entering the study, and the study was approved by the institutional ethics committee.

\section{Magnetic resonance imaging}

All participants were imaged with a 3.0-T magnetic resonance scanner (Signa Excite HDx 3.0, GE Medical Systems, Milwaukee, WI, USA) using three-dimensional fast imaging employing steady-state acquisition (3D FIESTA) and diffusion tensor imaging (DTI). An eight-channel head coil was used with foam padding and braces to restrict head motion. The following imaging protocols were used: (a) 3D FIESTA sequence with repetition time/echo time $(\mathrm{TR} / \mathrm{TE})=6.1 \mathrm{~ms} /$ $1.5 \mathrm{~ms}$, flip angle $=60^{\circ}$, field of view $=240 \mathrm{~mm} \times 240 \mathrm{~mm}$, matrix $=512 \times 512$, and two acquisitions; (b) DTI with a singleshot spin echo echo-planar imaging protocol, $\mathrm{TR} / \mathrm{TE}=10$, $000 \mathrm{~ms} / 96.6 \mathrm{~ms}$, field of view $=240 \mathrm{~mm} \times 240 \mathrm{~mm}$, matrix $=128 \times 128, b=0$ and $1000 \mathrm{~s} / \mathrm{mm}^{2}$ with diffusion gradients applied in 20 non-collinear directions, 19 slices of $2.4 \mathrm{~mm}$ without gap, and acquisition time $=5.12 \mathrm{~min}$.

\section{DTI data processing}

The trigeminal nerve was easily delineated in all participants by applying all MRI sequences. The diagnostic criteria [14] of
NVC were defined as no visible cerebrospinal fluid between the trigeminal root and its adjacent arteries on 3D FIESTA MRI (Fig. 4b1, c1). The mean sizes of DCA were measured at the site of NVC by 3D FIESTA. The original DTI data were processed with Functool software in an AW4.4 workstation (GE Medical Systems, Milwaukee, WI, USA) to generate fractional anisotropy (FA) (Fig. 4a2, b2, c2) maps, maximum $(\lambda 1)$, middle $(\lambda 2)$, and minimum $(\lambda 3)$ eigenvector maps (Fig. 1). We placed box-shaped regions of interest (ROIs) over the root entry zone of the trigeminal nerve to measure $\lambda 1, \lambda 2$, and $\lambda 3$ (Fig. 1). For each ROI (approximately $30 \mathrm{~mm}^{2}$ ), the $\mathrm{AD}$ and $\mathrm{RD}$ were calculated based on the following equations $[1,2,16-19]: \mathrm{AD}=\lambda 1, \mathrm{RD}=(\lambda 2+\lambda 3) / 2$

\section{Statistical analysis}

All statistical calculations were performed with SPSS 16 software (SPSS Inc., Chicago, IL, USA). The DCA and all diffusion metrics, including $\mathrm{AD}$ and $\mathrm{RD}$ were measured and calculated independently by two observers who were blinded to the clinical features and purpose of this study. For statistical analysis, we utilized the mean values from the two observers. Table 1 compares the mean of DCA between two groups (NVC with TN and without TN); AD and RD are compared between the three groups (NVC with TN, NVC without TN, and $\mathrm{HC}$ ) using a one-way analysis of variance (ANOVA) followed by Bonferroni's multiple comparison tests. Results are expressed as mean \pm SD (Table 1). $p$ values $<0.05$ were considered statistically significant. The correlation analysis between DCA and diffusion metrics (AD and RD) was ere also performed.

\section{Results}

\section{Offending arteries and compression site of NVC}

Based on the 3D FIESTA MRI findings, the trigeminal nerve could be easily delineated on both sides in all the three groups (NVC with TN, NVC without TN, and HC). All HC did not present with NVC. In more than half of the NVC patients with and without TN $(82 / 100,82 \%)$, contact with the superior cerebellar artery (SCA) was identified, other patients had nerve contact with other arteries including the anterior inferior cerebellar artery (AICA) $(14 / 100,14 \%)$, vertebral artery (VA) $(2 / 100,2 \%)$, and posterior inferior cerebellar artery (PICA) $(2 / 100,2 \%)$. In most of the NVC patients with TN, the proximal part of the arteries caused compression (49/50, $98 \%$ ), and only in one patient the distal part of VA caused compression $(1 / 50,2 \%)$; while in NVC patients without TN, compression was induced by the distal part of the arteries (40/50 patients, $80 \%$ ), proximal part of the arteries $(3 / 50,6 \%)$, and artery branch $(7 / 50,14 \%)$. 
Fig. 1 We placed a box-shaped ROI over the root entry zone of the trigeminal nerves to measure $\lambda 1, \lambda 2$, and $\lambda 3$ in the maximum, middle, and minimum eigenvector maps, respectively. The ROI size of the left and right side was equivalent (approximately $30 \mathrm{~mm}^{2}$ )

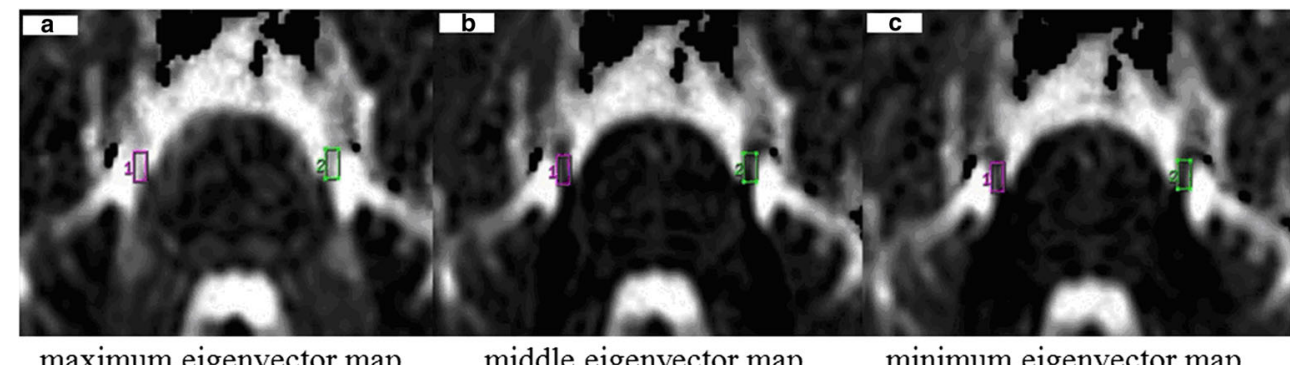

\section{DCA and diffusion metrics}

Table 1 summarizes the DCA and diffusion metrics of our study. The mean DCA $(1.58 \pm 0.34 \mathrm{~mm})$ was larger in NVC patients with TN than those NVC patients without TN $(0.89$ $\pm 0.29 \mathrm{~mm})$. Compared with the $\mathrm{NVC}$ without $\mathrm{TN}(\mathrm{RD}=1.17$ $\pm 0.13)$ and $\mathrm{HC}(\mathrm{RD}=1.15 \pm 0.24)$, the mean values of $\mathrm{RD}$ $(1.69 \pm 0.21)$ at the site of the NVC with TN were significantly increased; however, no significant changes of $\mathrm{AD}$ was found between the three groups $(\mathrm{AD}=2.91 \pm 0.10,2.91 \pm 0.11,2.89$ \pm 0.07 , respectively).

\section{Correlation between DCA and diffusion metrics}

The correlation analysis showed that DCA significantly and positively correlated with RD in NVC patients with and without TN (RD and DCA: $r=0.830, p=0.000$; Fig. 2). The correlation between DCA and AD were also calculated, which showed no significant correlation (AD and DCA: $r=0.178$, $p=0.077$, Fig. 3).

\section{Discussion}

\section{Compression site and DCA in NVC with and without TN}

TN is an unbearable pain syndrome caused by several mechanisms. Compression of trigeminal nerves by the arteries is considered one of the primary causes $[3,6]$. TN is also considered to be related to the size of DCA. The aim of this study was to assess the mean sizes of DCA between NVC with and without TN, and to investigate microstructural tissue changes of trigeminal nerve using $\mathrm{AD}$ and RD of MRI. To further explore the relationship between the DCA and TN, correlation analyses were performed between DCA and diffusion metrics (AD and RD).

In our study, compression by the proximal part of the arteries was observed in most of the NVC patients with TN $(49 / 50$, $98 \%$ ), while compression by the distal part of the arteries was observed in most of the NVC patients without TN $(40 / 50,80 \%)$. 3D FIESTA can show the diameter of compression arteries clearly, the mean DCA with TN was $1.58 \pm 0.34 \mathrm{~mm}$, and mean DCA without TN was $0.89 \pm 0.29 \mathrm{~mm}$. The DCA with TN was significantly larger than that without TN (Table 1). The large DCA may have an effect on the occurrence of TN.

\section{Diffusion metrics in NVC with and without TN}

We showed that AD and RD of DTI are useful in detecting microstructural changes of trigeminal nerve in the three groups. A study by Liu et al. [12] has suggested that specific diffusion metrics of $\mathrm{AD}$ and RD may be used to specifically assess trigeminal nerve change. $\mathrm{AD}=\lambda 1$, where $\lambda 1$ represents the water diffusivity parallel to the axonal fibers, and therefore reflects axonal changes of the cerebral white matter (WM). $\mathrm{RD}=(\lambda 2+\lambda 3) / 2$, where $\lambda 2$ and $\lambda 3$ represent water diffusion in the planes orthogonal to the long axis of the axon, which thus reflects myelin changes (such as demyelination and remyelination) of cerebral $\mathrm{WM}$, and eigenvalues $(\lambda 1, \lambda 2$, and $\lambda 3)$ are the magnitudes of eigenvectors $[1,17,19]$.

Table 1 summarizes the detailed results of our study. Compared with $\mathrm{NVC}$ without $\mathrm{TN}(\mathrm{RD}=1.17 \pm 0.13)$ and $\mathrm{HC}$ $(\mathrm{RD}=1.15 \pm 0.24)$, the mean values of $\mathrm{RD}(1.69 \pm 0.21)$ at the site of the NVC with TN were significantly increased; however, no significant change of $\mathrm{AD}$ was found between
Table 1 Mean values of $\mathrm{DCA}, \mathrm{AD}$, and RD in the three groups (NVC with $\mathrm{TN}, \mathrm{NVC}$ without TN, and $\mathrm{HC}$ )

\begin{tabular}{lllllll}
\hline Variable & $\begin{array}{l}\text { NVC with } \\
\text { TN }\end{array}$ & $\begin{array}{l}\text { NVC without } \\
\text { TN }\end{array}$ & HC & $\begin{array}{l}\text { NVC with TN vs. } \\
\text { NVC without TN }\end{array}$ & $\begin{array}{l}\text { NVC with } \\
\text { TN vs. HC }\end{array}$ & $\begin{array}{l}\text { NVC without } \\
\text { TN vs. HC }\end{array}$ \\
\hline $\mathrm{DCA}(\mathrm{mm})$ & $1.58 \pm 0.34$ & $0.89 \pm 0.29$ & - & $p=0.00$ & - & - \\
$\mathrm{RD}\left(\times 10^{-3} \mathrm{~mm}^{2} / \mathrm{s}\right)$ & $1.69 \pm 0.21$ & $1.17 \pm 0.13$ & $1.15 \pm 0.24$ & $p=0.00$ & $p=0.00$ & $p=0.34$ \\
$\mathrm{AD}\left(\times 10^{-3} \mathrm{~mm}^{2} / \mathrm{s}\right)$ & $2.91 \pm 0.10$ & $2.91 \pm 0.11$ & $2.89 \pm 0.07$ & $p=0.78$ & $p=0.16$ & $p=0.27$ \\
\hline
\end{tabular}

$D C A$ diameter of compression arteries, $A D$ axial diffusivity, $R D$ radial diffusivity, $N V C$ neurovascular compression, $T N$ trigeminal neuralgia, $H C$ healthy controls

$p<0.05$ was considered significant 
Fig. 2 Correlation analyses between RD and DCA in NVC patients with and without TN $(n=100)$. Note: $R D$ radial diffusivity $\left(\times 10^{-3} \mathrm{~mm}^{2} / \mathrm{s}\right), D C A$ diameter of compression arteries (mm), $N V C$ neurovascular compression, $T N$ trigeminal neuralgia

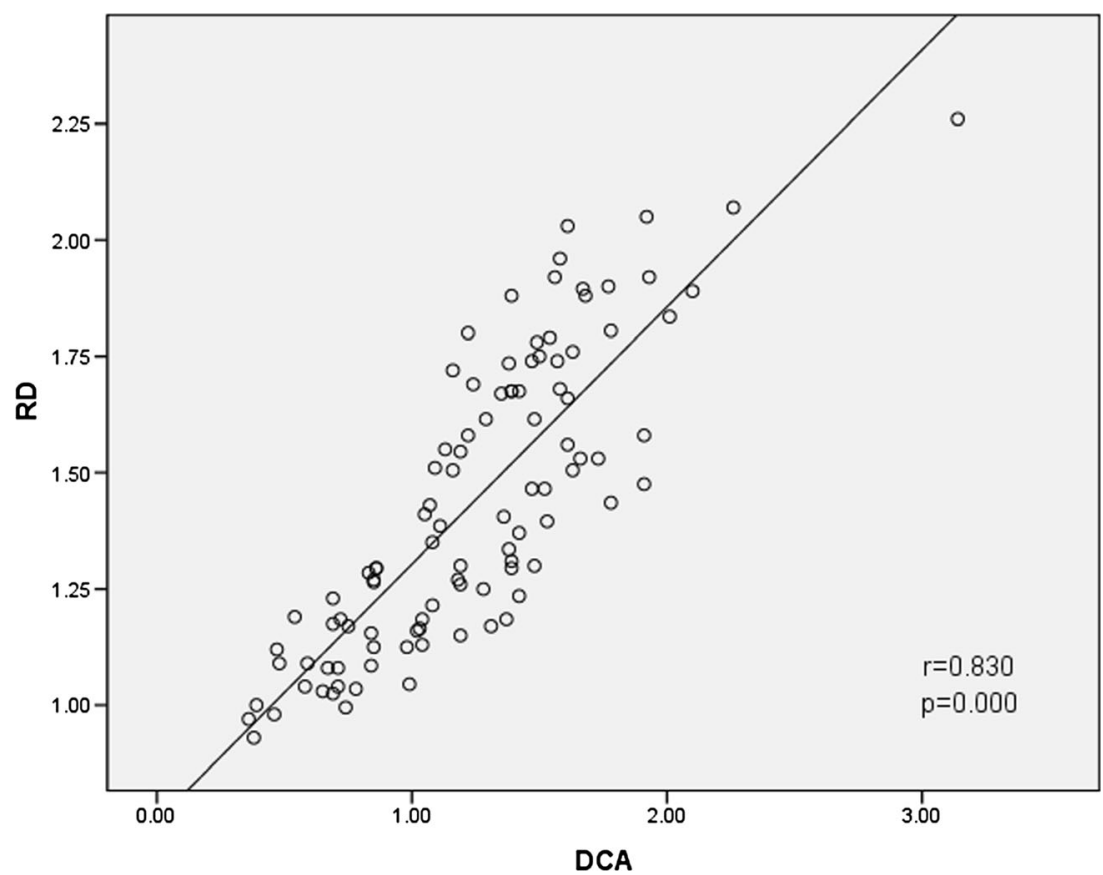

the three groups $(\mathrm{AD}=2.91 \pm 0.10,2.91 \pm 0.11,2.89 \pm 0.07$, respectively). Increased RD indicates that there is focal demyelination at the compressed sides of NVC with TN. Compared with $\mathrm{HC}$, no significant difference in RD means there is no focal demyelination at the compressed sides of NVC without TN.

\section{Correlation between DCA and diffusion metrics}

Liu et al. [12] reported that, compared with $\mathrm{HC}$ and the uncompressed side, the compressed side showed significantly increased RD and unchanged AD. Their observations suggested that the primary TN pathology is the focal demyelination of the sensory axons at the site of the NVC, and they further indicated that demyelination without significant axonal injury is an essential pathological basis of the compressed trigeminal nerve. $\mathrm{AD}$ and $\mathrm{RD}$ have been used to characterize trigeminal nerve damage from NVC. Our correlation analysis showed that DCA significantly and positively correlated with $\mathrm{RD}$ in NVC patients with and without TN (RD and DCA: $r=0.830, p=0.000$; Fig. 2). Large arteries are more likely to cause higher $\mathrm{RD}$ and more easily to make trigeminal nerve
Fig. 3 Correlation analyses between $\mathrm{AD}$ and DCA in NVC patients with and without TN $(n=100)$. Note: $A D$ axial diffusivity $\left(\times 10^{-3} \mathrm{~mm}^{2} / \mathrm{s}\right), D C A$ diameter of compression arteries (mm), $N V C$ neurovascular compression, $T N$ trigeminal neuralgia

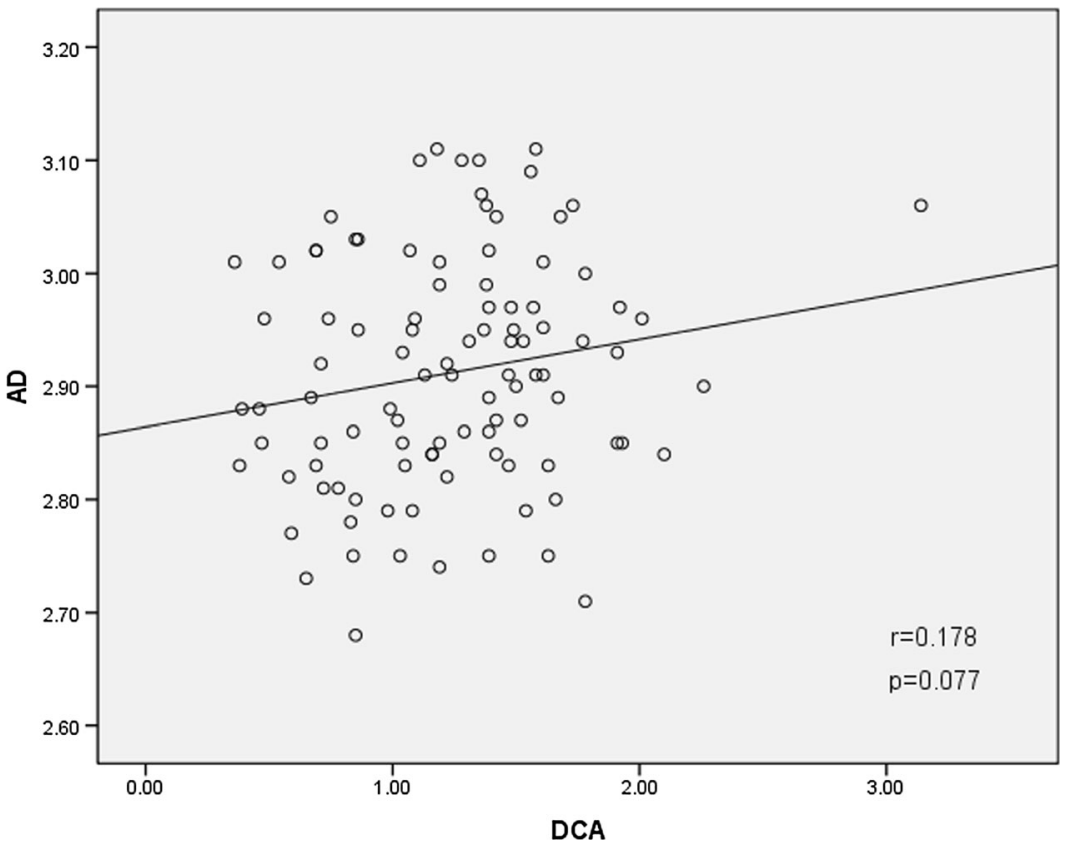




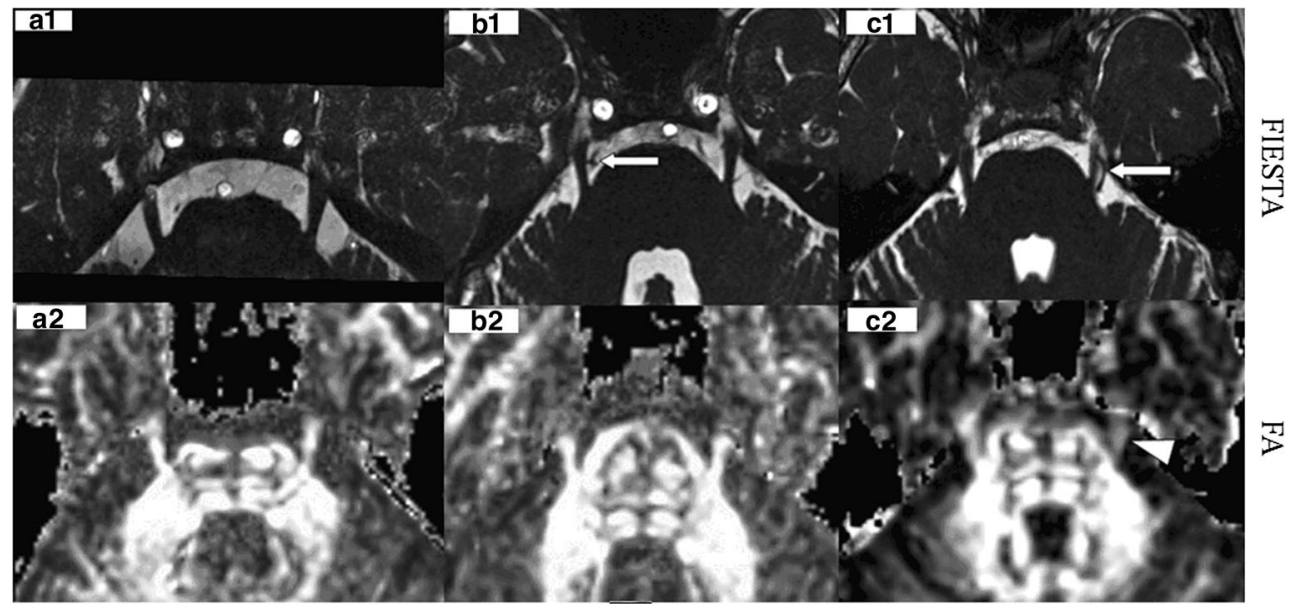

Fig. 4 a1 3D FIESTA MR image showing a 53-year-old man (HC without NVC). a2 fractional anisotropy image showing same signal in both left and right trigeminal nerve; b1 an artery branch (arrow) (DCA: $0.70 \mathrm{~mm}$ ) compressing the right trigeminal nerve root in a 52-year-old asymptomatic woman (NVC without TN). b2 fractional anisotropy image showing same signal in both left and right trigeminal nerves; $\mathbf{c 1}$ the

demyelination, while small arteries are not. Some studies [4, $5,10,12]$ showed that trigeminal nerve demyelination is prevalent in TN patients. Demyelination causes destruction of the trigeminal nerve integrity, which leads to TN. Hence TN may be the result of chronic and long-term physical compression of the large diameter of the arteries. The correlation between DCA and AD were also calculated, which showed no significant correlation (AD and DCA: $r=0.178, p=0.077$; Fig. 3), NVC might rarely cause trigeminal axon damage.

\section{Possible causes of TN with larger DCA}

According to Kamiguchi et al. [7], NVC is caused by elongation, tortuosity, dilatation, or variant arteries. Regarding vascular compression of the trigeminal nerve, some patients may have symptoms, while others are asymptomatic $[5,11,13]$. Why? There are many reasons for answering these questions. Some studies reported $[4,5,10]$ that $\mathrm{TN}$ is likely a result of chronic vascular compression. Other study suggested that symptoms may be related to the degree of vascular compression, the more serious the trigeminal nerve is depressed, the greater is the presence of clinical symptoms [3]. However, no study has shown whether the size of DCA has some influence on TN. Miller et al. [13] reported patients with TN with NVC that is, on average, more proximal to the nerve than patients without facial pain. Our study showed that the NVC of TN patients was often from the proximal part of the arteries. The DCA of proximal arteries is bigger than the distal ones. The size of DCA at the site of NVC may influence the severity of a neurovascular conflict [8]. Leal PR et al. [9] suggested that atrophic changes in trigeminal nerves, which significantly correlated with the severity of the NVC. Our study showed that DCA significantly and positively correlated with RD in NVC proximal SCA (arrow) (DCA: $1.56 \mathrm{~mm}$ ) compressing the left trigeminal nerve root in a 58-year-old TN woman (NVC with TN). c2 fractional anisotropy image showing hypointensity signal in the left nerve (arrowhead) compared with the opposite side. Note: $H C$ healthy controls, $N V C$ neurovascular compression, $D C A$ diameter of compression arteries, $T N$ trigeminal neuralgia, $S C A$ superior cerebellar artery

patients with and without TN (Fig. 2). Increased RD indicates that there is focal demyelination at the compressed sides of NVC with TN [12]. Due to its incessant pulsations, the large DCA (Fig. 4c1), such as the proximal part of the offending arteries, may have sufficient and chronic physical pressure on the trigeminal root that causes chronic injury to the myelin sheath and leads to trigeminal nerve focal demyelination and loss of anisotropy. In our study, the fractional anisotropy image shows hypointensity signal in the compressed nerve compared with the opposite side (Fig. 4c2), and ultimately leads to $\mathrm{TN}$, while smaller DCA such as the distal part of the offending arteries and artery branch (Fig. 4b1) may therefore have insufficient pressure on the trigeminal nerve, which may not cause focal demyelination and in turn does not lead to TN; there is no signal intensity change in fractional anisotropy image (Fig. 4b2). According to our study results, the larger the DCA in the NVC, the more are the chances of suffering from $\mathrm{TN}$. The larger DCA might play an important role in TN.

\section{Conclusions}

The mean DCA in NVC patients with TN were larger than those without TN. The mean values of RD at the site of the NVC with TN were significantly increased than both NVC without TN and HC. However, no significant changes of $\mathrm{AD}$ were found between the three groups. Our study also showed that DCA significantly and positively correlated with RD in NVC patients with and without TN. Our study showed that larger DCA may increase the chance of trigeminal nerve demyelination, and may be a possible facilitating factor for $\mathrm{TN}$. 
Acknowledgments We thank Xingyue HU, Professor of Department of Neurology, Sir Run Run Shaw Hospital, School of Medicine, Zhejiang University, Hangzhou, China, for his help during the preparation of this manuscript. This work was also technically supported by GE Healthcare.

Compliance with ethical standards This study was approved by the institutional ethics committee.

Funding This study was supported by Zhejiang Provincial Natural Science Foundation of China (No. LY14H180001). The sponsor had no role in the design or conduct of this research.

\section{Conflicts of interest None.}

Informed consent Written informed consent was obtained from each participant before entering the study.

Open Access This article is distributed under the terms of the Creative Commons Attribution 4.0 International License (http:// creativecommons.org/licenses/by/4.0/), which permits unrestricted use, distribution, and reproduction in any medium, provided you give appropriate credit to the original author(s) and the source, provide a link to the Creative Commons license, and indicate if changes were made.

\section{References}

1. Boretius S, Escher A, Dallenga T, Wrzos C, Tammer R, Brück W, Nessler S, Frahm J, Stadelmann C (2012) Assessment of lesion pathology in a new animal model of MS by multiparametric MRI and DTI. Neuroimage 59:2678-2688

2. Burgmans S, Gronenschild EH, Fandakova Y, Shing YL, van Boxtel MP, Vuurman EF, Uylings HB, Jolles J, Raz N (2011) Age differences in speed of processing are partially mediated by differences in axonal integrity. Neuroimage 55:1287-1297

3. Chun-Cheng Q, Qing-Shi Z, Ji-Qing Z, Zhi-Gang W (2009) A single-blinded pilot study assessing neurovascular contact by using high-resolution MR imaging in patients with trigeminal neuralgia. Eur J Radiol 69:459-463

4. Erbay SH, Bhadelia RA, O'Callaghan M, Gupta P, Riesenburger R, Krackov W, Polak JF (2006) Nerve atrophy in severe trigeminal neuralgia: noninvasive confirmation at MR imaging-initial experience. Radiology 238:689-692

5. Ha SM, Kim SH, Yoo EH, Han IB, Shin DA, Cho KG, Chung SS, Park YS (2012) Patients with idiopathic trigeminal neuralgia have a sharper-than-normal trigeminal-pontine angle and trigeminal nerve atrophy. Acta Neurochir (Wien) 154:1627-1633

6. Jannetta PJ, McLaughlin MR, Casey KF (2005) Technique of microvascular decompression. Technical note. Neurosurg Focus 18:E5
7. Kamiguchi H, Ohira T, Ochiai M, Kawase T (1997) Computed tomographic analysis of hemifacial spasm: narrowing of the posterior fossa as a possible facilitating factor for neurovascular compression. J Neurol Neurosurg Psychiatry 62:532-534

8. Krzyżewski RM, Stachura MK, Stachura AM, Rybus J, Tomaszewski KA, Klimek-Piotrowska W, Brzegowy P, Urbanik A, Walocha JA (2014) Variations and morphometric analysis of the proximal segment of the superior cerebellar artery. Neurol Neurochir Pol 48:229-235

9. Leal PR, Barbier C, Hermier M, Souza MA, Cristino-Filho G, Sindou M (2014) Atrophic changes in the trigeminal nerves of patients with trigeminal neuralgia due to neurovascular compression and their association with the severity of compression and clinical outcomes. J Neurosurg 120:1484-1495

10. Leal PR, Roch JA, Hermier M, Souza MA, Cristino-Filho G, Sindou M (2011) Structural abnormalities of the trigeminal root revealed by diffusion tensor imaging in patients with trigeminal neuralgia caused by neurovascular compression: a prospective, double-blind, controlled study. Pain 152:2357-2364

11. Lin W, Chen YL, Zhang QW (2014) Vascular compression of the trigeminal nerve in asymptomatic individuals: a voxelwise analysis of axial and radial diffusivity. Acta Neurochir (Wien) 156:577-780

12. Liu Y, Li J, Butzkueven H, Duan Y, Zhang M, Shu N, Li Y, Zhang Y, Li K (2013) Microstructural abnormalities in the trigeminal nerves of patients with trigeminal neuralgia revealed by multiple diffusion metrics. Eur J Radiol 82:783-786

13. Miller JP, Acar F, Hamilton BE, Burchiel KJ (2009) Radiographic evaluation of trigeminal neurovascular compression in patients with and without trigeminal neuralgia. J Neurosurg 110:627-632

14. Peker S, Dinçer A, Necmettin Pamir M (2009) Vascular compression of the trigeminal nerve is a frequent finding in asymptomatic individuals: 3-T MR imaging of 200 trigeminal nerves using 3D CISS sequences. Acta Neurochir (Wien) 151:1081-1088

15. Scrivani SJ, Mathews ES, Maciewicz RJ (2005) Trigeminal neuralgia. Oral Surg Oral Med Oral Pathol Oral Radiol Endod 100:527538

16. Seal ML, Yücel M, Fornito A, Wood SJ, Harrison BJ, Walterfang M, Pell GS, Pantelis C (2008) Abnormal white matter microstructure in schizophrenia: a voxelwise analysis of axial and radial diffusivity. Schizophr Res 101:106-110

17. Song SK, Yoshino J, Le TQ, Lin SJ, Sun SW, Cross AH, Armstrong RC (2005) Demyelination increases radial diffusivity in corpus callosum of mouse brain. Neuroimage 26:132-140

18. Xu J, Shimony JS, Klawiter EC, Snyder AZ, Trinkaus K, Naismith RT, Benzinger TL, Cross AH, Song SK (2013) Improved in vivo diffusion tensor imaging of human cervical spinal cord. Neuroimage 67:64-76

19. Zhong WJ, Guo DJ, Zhao JN, Xie WB, Chen WJ, Wu W (2012) Changes of axial and radial diffusivities in cerebral white matter led by normal aging. Diagn Interv Imaging 93:47-52 